\title{
“Ancestralization” of human pluripotent stem cells by multiplexed precise genome editing
}

Stephan Riesenberg*, Tomislav Maricic, Svante Pääbo

*corresponding author

Department of Evolutionary Genetics, Max Planck Institute for Evolutionary Anthropology 
We show that inactivation of the DNA-dependent protein kinase catalytic subunit (DNA-PKcs) results in a drastic increase in efficiency of precise genome editing with CRISPR enzymes in human stem cells, allowing up to $79 \%$ of chromosomes to carry an intended nucleotide substitution when a single genomic site is targeted. When three different genes are simultaneously targeted, $12 \%$ of the isolated cells carry the targeted amino acid-changing substitutions in homozygous forms. These substitutions represent the first step towards resurrecting the proteome ancestral to Neandertals and modern humans. DNA-PKes inactivation will greatly facilitate multiplexed precise genome editing in animal cells.

Genome sequences of our closest evolutionary relatives, Neandertals and Denisovans, allow the identification of novel genetic changes that are present in all or almost all humans today. These changes are of interest because some of them may be involved in phenotypes that made the cultural and technological developments associated with modern humans possible ${ }^{1}$. However, because the ancestral versions of such changes are absent or extremely rare in present-day humans, their physiological consequences cannot be easily studied, especially not in a homozygous form. Furthermore, it is likely that combinations of such mutations may be necessary to induce relevant phenotypes. One way to study such changes is to introduce the ancestral genetic variants in human induced pluripotent stem cells (iPSCs) which can then be differentiated into various cell types ${ }^{2}$ or used to generate organoids ${ }^{3,4}$. Unfortunately, this approach is limited by the inability to introduce multiple precise genetic changes in mammalian cells. To overcome this limitation, we developed an approach to simultaneously introduce multiple nucleotide substitutions in a homozygous form in human stem cells.

Genome editing uses clustered regularly interspaced short palindromic repeats (CRISPR) nucleases, or other nucleases ${ }^{5}$, to introduce targeted double-strand breaks (DSBs) in a genome. These are then repaired by two competing cellular pathways: non-homologous end-joining (NHEJ) or homology-directed repair (HDR). Because NHEJ often introduces deletions or insertions (indels) it is often used to inactivate genes. In contrast, HDR utilizes a sister chromatid to repair the chromosome carrying a DNA break. This activity can be used for precise genome editing (PGE) if exogenous DNA carrying desired mutations is provided as a donor. However, NHEJ is typically more efficient than HDR, leading to low PGE efficiencies. As a result, multiplexed PGE has not been reported in animal cells while multiplexed introduction of insertions and deletions has been achieved. For example, eight alleles of five different genes have been inactivated in mouse embryonic stem cells by insertions or deletions ${ }^{6}$ and in a porcine immortalized cell line 62 copies of an endogenous retrovirus have been inactivated by the use of a two guide RNAs ${ }^{7}$. This shows that $D N A$ cleavage by CRISPR enzymes is not the ratelimiting step for genome editing. Rather, the higher efficiency of NHEJ relative to HDR limits the ability to introduce precise substitutions. Several studies have therefore tried to inhibit NHEJ in order to increase the efficiencies of PGE, for example by blocking the synthesis of NHEJ proteins with siRNAs, by the expression of adenovirus proteins that degrade proteins necessary for NHEJ ${ }^{8}$, and by using small 
molecules that block NHEJ ${ }^{9,10}$. Others have tried to enhance HDR by cell cycle synchronization ${ }^{11,12}$ and improved DNA donor design ${ }^{13-15}$. A third approach that does not rely on HDR to introduce nucleotide substitutions is to use proteins that chemically induce nucleotide transitions but this allows only four out of 12 possible substitutions to be introduced ${ }^{16,17}$.

We focused our efforts to increase HDR and thus PGE on the DNA-dependent protein kinase (DNA-PK) complex, which consists of the subunits Ku70, Ku80 and the DNA-PK catalytic subunit (DNA-PKcs) and covers the DNA ends after a DSB is introduced (Fig. 1A) ${ }^{18}$. When bound to DNA ends, the DNA-PKcs undergoes autophosphorylation and recruits other proteins such as DNA ligase IV, XRCC4, and XLF ${ }^{18}$ which are necessary for canonical NHEJ ${ }^{19,20}$. If canonical NHEJ is compromised, a slower DNA-PKcs-independent alternative NHEJ pathway may take over ${ }^{21}$. Alternatively, HDR can be induced if a protein complex composed of Mre11, Rad50 and Nbs1 binds to the DNA ends. This results in the generation of 3' overhanging DNA ends, RAD51 nucleoprotein filament formation, and annealing of donor DNA that serve as template for DNA synthesis leading to the faithful repair of the DSB ${ }^{18,22}$.

When bound to DSBs, the $\sim 470 \mathrm{kDa}$ DNA-PKcs protein undergoes conformational changes induced at least in part by autophosphorylation ${ }^{23,24}$ as well as by other related kinases ${ }^{25,26}$ at more than 60 phosphorylation sites ${ }^{20,27,28}$. When phosphorylated, some sites activate the kinase activity of DNAPKcs, while others disengage c-NHEJ and inactive the kinase activity of DNA-PKcs (Fig. 1B). In 2008, it was shown that a lysine to arginine mutation at position 3753 near the ATP binding site in the DNAPKcs protein (KR) abolishes its kinase activity ${ }^{22,29}$ in Chinese hamster ovary cells. This increased HDR to levels 2- to 3-fold above those seen when the DNA-PKcs gene was completely inactivated ${ }^{29}$.

To achieve high efficiency of targeted DSBs with few off-target DSBs we first generated an iPSC line carrying a doxycycline inducible Cas9 nickase (iCRISPR-Cas9n) as described by Gonzalez et al. ${ }^{30,31}$. We then used this line to introduce a mutation in the PRKDC gene that causes the K3753R mutation in the DNA-PKcs protein ${ }^{29}$. We tested the efficiency with which nucleotide substitutions can be precisely introduced in DNA-PKcs KR (KR) and wildtype DNA-PKcs cells using three substitutions that cause amino acid changes that are fixed among present-day humans but occur in the ancestral, apelike state in the Neandertals and Denisovan genomes. These substitutions occur in the genes CALD1, KATNA1 and SLITRK1 that are involved in neurite outgrowth. Changes in them may therefore affect connectivity or neuronal circuitry in the human brain.

We designed RNA guides for double nicking ${ }^{30}$ and single-stranded DNA donors carrying the mutations reverting the targeted amino acids to the ancestral states, namely V671I, A343T and A330S, in CALD1, KATNA1 and SLITRK1, respectively. Each donor also carried silent "blocking" mutations to prevent recutting of the target once edited. When editing each gene independently, HDR efficiencies were 2.5 -fold ( $36 \%$ edited chromosomes), 4.1 -fold ( $79 \%$ e.c.), and 7.8 -fold (51\% e.c.) higher for CALD1, KATNA1 and SLITRK1, respectively, in the KR cells than the wild type cells (Fig. 2A). This 
corresponds to a shift of the ratio of HDR to NHEJ from $0.23,0.29$ and 0.08 to $3.49,25.4$, and 2.60, respectively.

To test if the increase in HDR relative to NHEJ was dependent on cellular production of Cas9 nickase from an endogenous inducible gene, we introduced recombinant Cas9 or Cpf1 protein, respectively, without the induction of the endogenous iCRISPR-Cas9n gene, into KR cells by electroporation and edited $C A L D 1$ to its ancestral state and also introduced nucleotide substitutions in the HPRT gene in separate experiments. The precise editing of CALD1 increased 4.8-fold and the editing of HPRT increased 8.3-fold (Fig. 2B), corresponding to a shift of the ratio of HDR/NHEJ from 0.45 to 12.8 and 0.08 to 7.1 , respectively. Thus, PGE is increased in the KR cells compared to wildtype cells regardless of which enzyme is used to induce DSBs.

Encouraged by the high HDR efficiencies in the KR cells, we electroporated the six guide RNAs and the three donor DNAs for CALD1, KATNA1 and SLITRK1 into KR cells after induction of Cas9 nickase expression. When scoring HDR as the presence of any of the nine intended mutations (two silent "blocking" mutations and one amino acid replacement in each gene), we achieved HDR efficiencies of $21 \%$ for CALD1, 32\% for KATNA1, and 34\% for SLITRK1 (Fig. 3A). We proceeded to isolate 33 cellular clones from the population of edited cells. Strikingly, 11 clones (33\%) carry at least one targeted nucleotide substitution (TNS) on both chromosomes without any insertions or deletions in any of the three genes (Fig. 3C, left panels). Four clones (12\%) carry all three 'ancestral' TNSs without any additional insertions or deletions, thus resulting in the three desired changes in a homozygous form (Fig. $3 \mathrm{C}$, right panels).

The results show that the inactivation of the kinase activity of the DNA-PKcs causes almost all DSBs induced by the CRSPR nuclease in the targeted genes to be repaired by HDR rather than NHEJ (Fig. 2 and 3) indicating that NHEJ is almost completely inactivated. One concern is that this may lead to genomic instability because double-stranded DNA breaks that occur spontaneously due to both endogenous and exogenous factors the cells cannot be repaired by NHEJ. Encouragingly, the DNAPKcs KR cells maintained a healthy karyotype with no chromosomal aberrations over 26 passages (Supplementary Fig. 1). In addition, the efficiency with which nucleotide substitutions can be introduced in these cells makes it possible to restore the normal function of the PRKDC gene once the desired changes have been introduced. We demonstrated this by editing the PRKDC gene back to its wildtype state and find that this "reverse" mutation was successfully introduced in $71 \%$ of chromosomes (Fig. 2A).

Notably, the incorporation of homozygous TNSs in all three genes is more than four-fold higher than expected by chance given the efficiencies of the introduction of substitutions when single genes are targeted. This suggests that KR cells that are "editing competent" will tend to be efficiently edited at multiple loci when presented with the relevant guide and donor nucleotides. Future work will determine the extent to which larger numbers of substitutions can be simultaneously introduced and further optimize the efficacy of multiplexed PGE. 
bioRxiv preprint doi: https://doi.org/10.1101/342311; this version posted June 8, 2018. The copyright holder for this preprint (which was not certified by peer review) is the author/funder, who has granted bioRxiv a license to display the preprint in perpetuity. It is made available under aCC-BY-NC-ND 4.0 International license.

Multiplexed PGE as described here is an advance in that it allows several substitutions to be introduced in a single round of editing. Because the lysine residue at position 3753 which we mutated in the KR cells is conserved among vertebrates (Fig. 1B) we expect that its function is also conserved so that the approach taken here is likely to be applicable not only in human but in many vertebrates. A single round of experiments as performed here where three homozygous nucleotide substitutions are introduced can be completed in two weeks. Thus, many substitutions can be introduced in a reasonable time frame. For example, it will allow the introduction of the 96 substitutions that revert 87 proteins to the primary sequences carried in the last common ancestor shared by modern humans and Neandertals 32 . Obviously, any other combinations of substitutions of medical, evolutionary or other interest could also be generated.

\section{Author contributions and acknowledgements}

S.R. conceived the idea and performed the experiments. S.R. and T.M and S.P. planned the experiments and wrote the paper. We would like to thank Antje Weihmann and Barbara Schellbach for DNA sequencing, and Heidrun Holland for karyotyping. This work was supported by the Max Planck Society and by the NOMIS foundation.

\section{Competing Interests}

A related patent application has been filed (EP 17203591.7).

\section{References}

$1 \quad$ Paabo, S. The human condition-a molecular approach. Cell 157, 216-226, doi:10.1016/j.cell.2013.12.036 (2014).

2 Robinton, D. A. \& Daley, G. Q. The promise of induced pluripotent stem cells in research and therapy. Nature 481, 295-305, doi:10.1038/nature10761 (2012).

3 Lancaster, M. A. \& Knoblich, J. A. Organogenesis in a dish: modeling development and disease using organoid technologies. Science 345, 1247125, doi:10.1126/science.1247125 (2014).

$4 \quad$ Mora-Bermudez, F. et al. Differences and similarities between human and chimpanzee neural progenitors during cerebral cortex development. Elife 5, doi:10.7554/eLife.18683 (2016).

5 Komor, A. C., Badran, A. H. \& Liu, D. R. CRISPR-Based Technologies for the Manipulation of Eukaryotic Genomes. Cell 169, 559, doi:10.1016/j.cell.2017.04.005 (2017).

$6 \quad$ Wang, H. et al. One-step generation of mice carrying mutations in multiple genes by CRISPR/Cas-mediated genome engineering. Cell 153, 910-918, doi:10.1016/j.cell.2013.04.025 (2013).

7 Yang, L. et al. Genome-wide inactivation of porcine endogenous retroviruses (PERVs). Science 350, 1101-1104, doi:10.1126/science.aad1191 (2015).

$8 \quad$ Chu, V. T. et al. Increasing the efficiency of homology-directed repair for CRISPR-Cas9induced precise gene editing in mammalian cells. Nat Biotechnol 33, 543-548, doi:10.1038/nbt.3198 (2015).

9 Maruyama, T. et al. Increasing the efficiency of precise genome editing with CRISPR-Cas9 by inhibition of nonhomologous end joining. Nat Biotechnol 33, 538-542, doi:10.1038/nbt.3190 (2015). 
bioRxiv preprint doi: https://doi.org/10.1101/342311; this version posted June 8, 2018. The copyright holder for this preprint (which was not certified by peer review) is the author/funder, who has granted bioRxiv a license to display the preprint in perpetuity. It is made available under aCC-BY-NC-ND 4.0 International license.

Robert, F., Barbeau, M., Ethier, S., Dostie, J. \& Pelletier, J. Pharmacological inhibition of DNAPK stimulates Cas9-mediated genome editing. Genome Med 7, 93, doi:10.1186/s13073-0150215-6 (2015).

Lin, S., Staahl, B. T., Alla, R. K. \& Doudna, J. A. Enhanced homology-directed human genome engineering by controlled timing of CRISPR/Cas9 delivery. Elife 3, e04766,

doi:10.7554/eLife.04766 (2014).

Yang, D. et al. Enrichment of G2/M cell cycle phase in human pluripotent stem cells enhances HDR-mediated gene repair with customizable endonucleases. Sci Rep 6, 21264, doi:10.1038/srep21264 (2016).

13 Richardson, C. D., Ray, G. J., DeWitt, M. A., Curie, G. L. \& Corn, J. E. Enhancing homologydirected genome editing by catalytically active and inactive CRISPR-Cas 9 using asymmetric donor DNA. Nat Biotechnol 34, 339-344, doi:10.1038/nbt.3481 (2016).

Renaud, J. B. et al. Improved Genome Editing Efficiency and Flexibility Using Modified Oligonucleotides with TALEN and CRISPR-Cas9 Nucleases. Cell Rep 14, 2263-2272, doi:10.1016/j.celrep.2016.02.018 (2016).

Lee, K. et al. Synthetically modified guide RNA and donor DNA are a versatile platform for CRISPR-Cas9 engineering. Elife 6, doi:10.7554/eLife.25312 (2017).

16 Komor, A. C., Kim, Y. B., Packer, M. S., Zuris, J. A. \& Liu, D. R. Programmable editing of a target base in genomic DNA without double-stranded DNA cleavage. Nature 533, 420-424, doi:10.1038/nature17946 (2016).

Gaudelli, N. M. et al. Programmable base editing of $A^{*} T$ to $G^{*} C$ in genomic DNA without DNA cleavage. Nature 551, 464-471, doi:10.1038/nature24644 (2017).

Dueva, R. I., George. Alternative pathways of non-homologous end joining (NHEJ) in genomic instability and cancer. Trans/ Cancer Res 2, 163-177 (2013).

Neal, J. A. et al. Inhibition of homologous recombination by DNA-dependent protein kinase requires kinase activity, is titratable, and is modulated by autophosphorylation. $\mathrm{Mol}$ Cell Biol 31, 1719-1733, doi:10.1128/MCB.01298-10 (2011).

Neal, J. A. et al. Unraveling the complexities of DNA-dependent protein kinase autophosphorylation. Mol Cell Biol 34, 2162-2175, doi:10.1128/MCB.01554-13 (2014). formation. Cytogenet Genome Res 104, 14-20, doi:10.1159/000077461 (2004). Shrivastav, M., De Haro, L. P. \& Nickoloff, J. A. Regulation of DNA double-strand break repair pathway choice. Cell Res 18, 134-147, doi:10.1038/cr.2007.111 (2008).

23 Meek, K., Lees-Miller, S. P. \& Modesti, M. N-terminal constraint activates the catalytic subunit of the DNA-dependent protein kinase in the absence of DNA or Ku. Nucleic Acids Res $\mathbf{4 0 ,}$ 2964-2973, doi:10.1093/nar/gkr1211 (2012).

24 Sibanda, B. L., Chirgadze, D. Y., Ascher, D. B. \& Blundell, T. L. DNA-PKcs structure suggests an allosteric mechanism modulating DNA double-strand break repair. Science 355, 520-524, doi:10.1126/science.aak9654 (2017).

25 Chen, B. P. et al. Ataxia telangiectasia mutated (ATM) is essential for DNA-PKcs phosphorylations at the Thr-2609 cluster upon DNA double strand break. J Biol Chem 282, 6582-6587, doi:10.1074/jbc.M611605200 (2007).

26 Yajima, H., Lee, K. J. \& Chen, B. P. ATR-dependent phosphorylation of DNA-dependent protein kinase catalytic subunit in response to UV-induced replication stress. Mol Cell Biol 26, 7520-7528, doi:10.1128/MCB.00048-06 (2006).

27 Dobbs, T. A., Tainer, J. A. \& Lees-Miller, S. P. A structural model for regulation of NHEJ by DNA-PKcs autophosphorylation. DNA Repair (Amst) 9, 1307-1314, doi:10.1016/j.dnarep.2010.09.019 (2010).

28 Hornbeck, P. V. et al. PhosphoSitePlus: a comprehensive resource for investigating the structure and function of experimentally determined post-translational modifications in man and mouse. Nucleic Acids Res 40, D261-270, doi:10.1093/nar/gkr1122 (2012).

29 Shrivastav, M. et al. DNA-PKcs and ATM co-regulate DNA double-strand break repair. DNA Repair (Amst) 8, 920-929, doi:10.1016/j.dnarep.2009.05.006 (2009).

30 Shen, B. et al. Efficient genome modification by CRISPR-Cas9 nickase with minimal off-target effects. Nat Methods 11, 399-402, doi:10.1038/nmeth.2857 (2014).

31 Gonzalez, F. et al. An iCRISPR platform for rapid, multiplexable, and inducible genome editing in human pluripotent stem cells. Cell Stem Cell 15, 215-226, doi:10.1016/j.stem.2014.05.018 (2014).

32 Prufer, K. et al. The complete genome sequence of a Neanderthal from the Altai Mountains. Nature 505, 43-49, doi:10.1038/nature12886 (2014). 
bioRxiv preprint doi: https://doi.org/10.1101/342311; this version posted June 8,2018 . The copyright holder for this preprint (which was not certified by peer review) is the author/funder, who has granted bioRxiv a license to display the preprint in perpetuity. It is made available under aCC-BY-NC-ND 4.0 International license.

Kent, W. J. et al. The human genome browser at UCSC. Genome Res 12, 996-1006, doi:10.1101/gr.229102. Article published online before print in May 2002 (2002).

\section{Figures}

A

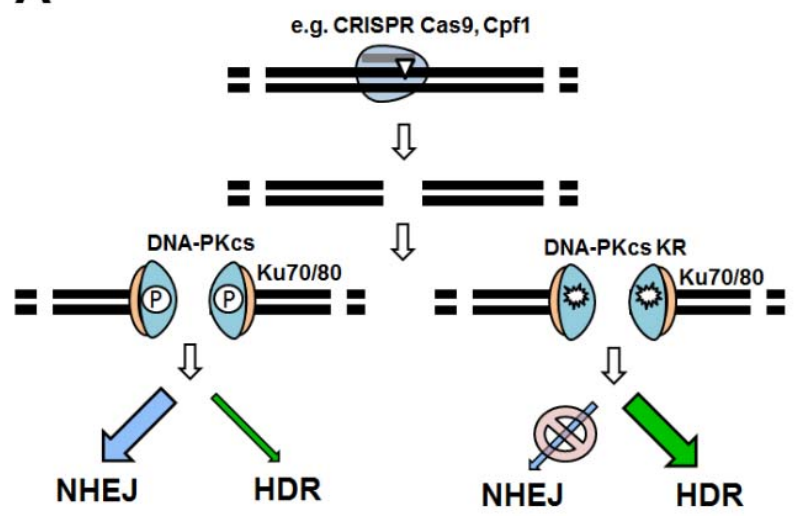

B

3753

E H P F L V K G G E D R Q Human EHPFLVKGGEDLRQ Rhesus

E Y P F L V K G G E D L R Q Chinese hamster EYPFLVKGGEDLRQ Mouse

EYPFLVKGGEDLRQ Dog

EYPFLVKGGEDLRQ Elephant

EHPFLVKGGEDLRQ Chicken

D Y P F L K G G E D R Q Zebrafish

Fig.1: Catalytically inactive DNA-PKcs (K3753R) promotes HDR and blocks NHEJ. (A) After a double strand break (DSB) induced by e.g. CRISPR-Cas9 or Cpf1 (light blue with gray gRNA), DNA ends are covered by Ku70/80 (orange), followed by binding of DNA-PKcs (cyan blue), both constituting a DNA-PK complex. Autophosphorylation of DNA-PKcs leads to recruitment and activation of downstream NHEJ proteins. If DNA-PKcs is catalytically inactivated (e.g. by the K3753R mutation), autophosphorylation cannot take place and the NHEJ pathway is be blocked ${ }^{22,29}$ leading to preferential HDR repair of the DSB. (B) The amino acid sequence of DNA-PKcs around K3753 is conserved among vertebrates (C) ${ }^{33}$.

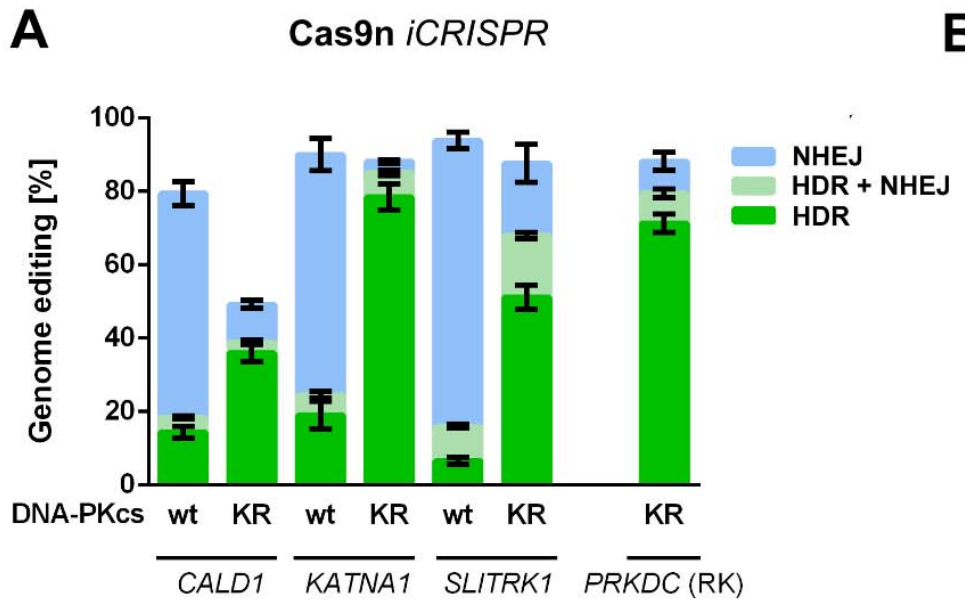

B Cas9 protein

Cpf1 protein
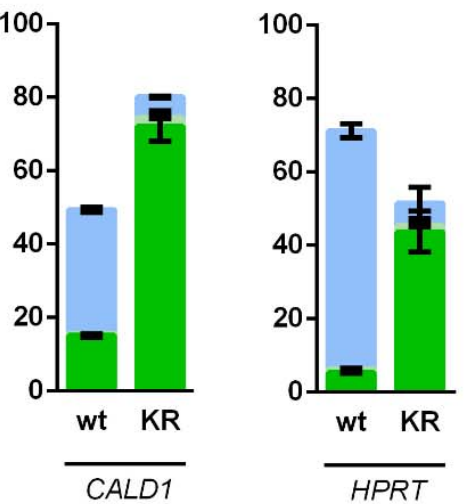

Fig. 2: Catalytically inactive DNA-PKcs (KR) leads to increased precise genome editing. (A) The frequency of NHEJ and HDR in the editing of the genes CALD1, KATNA1, SLITRK1 and PRKDC using Cas9n double nicking. (B) Editing of CALD1 with Cas9 nuclease and HPRT with Cpf1 nuclease. HDR, HDR + NHEJ, and NHEJ are indicated in green, light green and blue, respectively. Error bars show standard deviation of replicate experiments. 

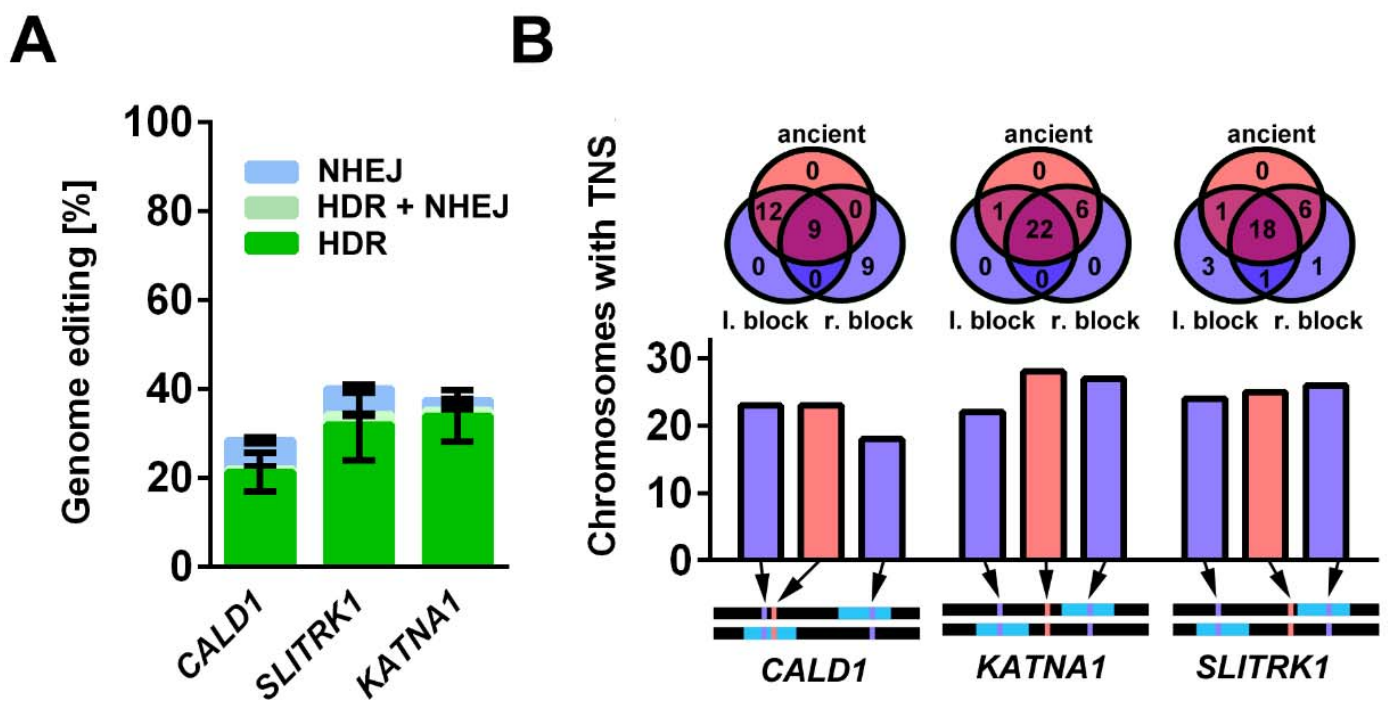

C
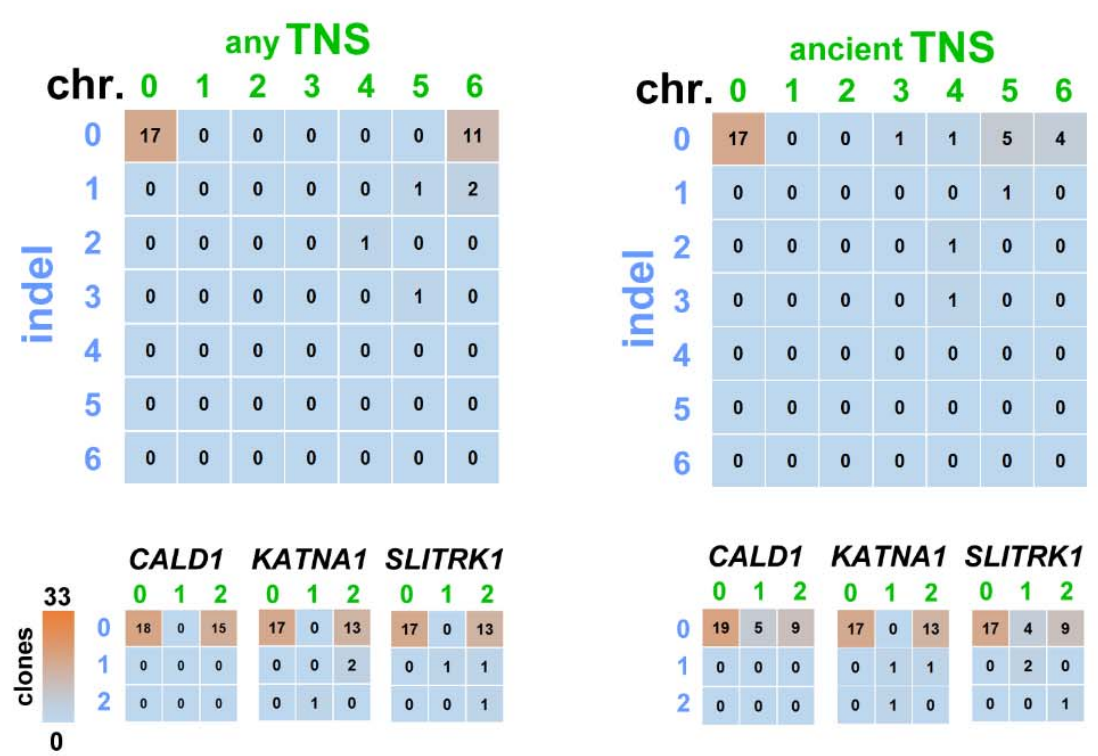

Fig. 3: Multiplexed precise genome editing of CALD1, KATNA1, and SLITRK1. (A) KR cells were electroporated with the guide RNAs and DNA donors for the three genes. The frequency of HDR, HDR + NHEJ, and NHEJ for each gene is given. Error bars show the standard deviation. (B) Venn diagrams illustrate how often targeted nucleotide substitutions (TNS), which are the left and right blocking mutations (purple) and 'ancient' missense mutations (pink), were observed in the chromosomes for each gene. Blocking mutations far from the other mutations (as the right one in CALD1) often occur in the absence of the other mutations. (C) Heat maps of genome editing events (TNS and/or indels) in chromosomes carrying CALD1, KATNA1, and SLITRK1 of 33 analyzed cellular clones are shown. Most clones either remain wildtype or are precisely edited for all three genes. Above all three genes (six chromosomes) with at least one TNS regardless if blocking mutation or 'ancient' mutation (left) or with at least the 'ancient' mutations (right). Below, the data for each gene (two chromosomes) is shown. An overview of mutations introduced together in CALD1, KATNA1, and SLITRK1 for each of the 33 clones is in Supplementary Table 2. 


\section{Methods (in Supplementary Materials)}

\section{Cell culture}

A human iPS line (409-B2, female, Riken BioResource Center) was used to create an iCRISPR-Cas9n line as described by Gonzalez et al. ${ }^{31}$ (GMO permit AZ 54-8452/26). The Puro-Cas9 donor was subjected to site-directed mutagenesis with the Q5 mutagenesis kit to introduce the D10A mutation (New England Biolabs, E0554S). Mutagenesis primers were from IDT (Coralville, USA) and are shown in Supplementary Table 1. Cells were grown on Matrigel Matrix (Corning, 35248) at $37^{\circ} \mathrm{C}$ in a humidified incubator with $5 \% \mathrm{CO}_{2}$ in mTeSR1 medium (StemCell Technologies, 05851) with supplement (StemCell Technologies, 05852) that was replaced daily. At $\sim 80 \%$ confluency, cells were dissociated using EDTA (VWR, 437012C) and diluted 1:1 in medium supplemented with $10 \mu \mathrm{M}$ Rhoassociated protein kinase (ROCK) inhibitor Y-27632 (Calbiochem, 688000) for one day after replating.

\section{Design of gRNAs and single stranded DNA donors}

To edit the genes CALD1, KATNA1 and SLITRK1 we used two gRNAs in tail-to-tail orientation with a small distance of gRNA pairs, and a cleavage efficiency above 45 (percentile rank score) as predicted by the sgRNA scorer 1.0 tool ${ }^{34}$. The single stranded DNA donors contained the desired mutation close to the middle of the sequence between the nicks as well as silent blocking mutations in each gRNA target close to the PAM to prevent re-cutting. At least 30nt homology arms were upstream or downstream of the respective nick site. For Cas9 nuclease cleavage, the gRNA of the CALD1 nickase gRNA pair that cuts closer to the desired mutation was used together with a 90nt single stranded DNA donor centered on the desired mutation and containing a blocking mutation. For Cpf1 cleavage, the positive control gRNA for HPRT from IDT (Coralville, USA) was used, together with a 90nt single stranded DNA donor with a blocking mutation near the PAM site and an additional mutation near the cut. RNAs and single stranded DNA donors were ordered from IDT (Coralville, USA) and are shown in Supplementary Table 1. The drop in multiplexed PGE efficiency from 33\% for the introduction of at least one of the substitutions for each gene to $12 \%$ for all three missense substitutions (Fig. 3C) is mostly due to the design of the $C A L D 1$ donor, where the right "blocking" mutation is further away from the "ancestral" mutation than in the other guide RNAs, causing this substitution to be the only one incorporated in $30 \%$ of CALD1 TNS-positive chromosomes (Fig. 3B).\#

\section{Lipofection of oligonucleotides}

409-B2 iCRISPR-Cas9n hiPSCs were incubated in medium containing $2 \mu \mathrm{g} / \mathrm{ml}$ doxycycline (Clontech, 631311) three days (four days for multiplexing) prior to lipofection. Lipofection was done using the altCRISPR protocol (IDT) at a final concentration of 7.5nM of each gRNA and 10nM of the DNA donors. In brief, $0.75 \mu 1$ RNAiMAX (Invitrogen, 13778075) and the respective oligonucleotides were separately

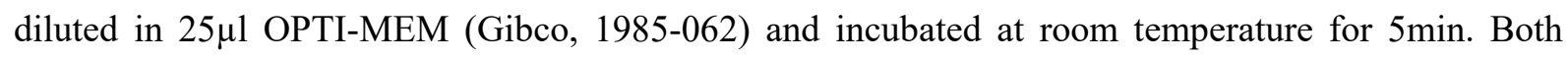
dilutions were mixed to yield $50 \mu 1$ of OPTIMEM including RNAiMAX, gRNAs and single stranded 
DNA donors. The lipofection mix was incubated for 20-30min at room temperature. Cells were dissociated using EDTA for $5 \mathrm{~min}$ and counted using the Countess Automated Cell Counter (Invitrogen). The lipofection mix, $100 \mu 1$ containing 25.000 dissociated cells in mTeSR1 supplemented with Y-27632, and $2 \mu \mathrm{g} / \mathrm{ml}$ doxycycline were put in one well of a 96 well covered with Matrigel Matrix (Corning, 35248). Media was exchanged to regular mTeSR1 media after 24 hours. We attempted multiplexed editing of three genes using lipofection, but achieved 3-10\% HDR for a gene at best (Supplementary Fig. 2).

\section{Oligonucleotide and ribonucleoprotein electroporation}

The recombinant Streptococcus pyogenes Cas9 protein, Acidaminococcus sp. BV3L6 Cpf1 protein and respective electroporation enhancer were from IDT (Coralville, USA) and electroporation was done using the manufacturer's protocol, except for the following alterations. Nucleofection was done using the B-16 program of the Nucleofector 2b Device (Lonza) in cuvettes for $100 \mu 1$ Human Stem Cell nucleofection buffer (Lonza, VVPH-5022), containing 1 million cells, 78pmol electroporation enhancer, 160pmol of each gRNA (crRNA/tracR duplex for Cas9 and crRNA for Cpf1), 200pmol of each single stranded DNA donor, and 252pmol Cas9 or Cpf1. For multiplexing, only gRNAs and single stranded DNA donors were electroporated, since a Cas9n expressing iCRISPR-Cas9n hiPSC line was used. Cells were counted using the Countess Automated Cell Counter (Invitrogen). 90 percent of the electroporated cells were plated for bulk genotype analysis and 10 percent were plated in a separate 6 well to give rise to colonies derived from a single cell (clones) for which the media was supplemented with Rhoassociated protein kinase (ROCK) inhibitor Y-27632 for three days post-electroporation. After at least seven days colonies were picked for following propagation and DNA isolation.

\section{Illumina library preparation and sequencing}

At least three days after transfection cells were dissociated using Accutase (SIGMA, A6964), pelleted, and resuspended in $15 \mu 1$ QuickExtract (Epicentre, QE0905T). Incubation at $65^{\circ} \mathrm{C}$ for $10 \mathrm{~min}, 68^{\circ} \mathrm{C}$ for $5 \mathrm{~min}$ and finally $98^{\circ} \mathrm{C}$ for $5 \mathrm{~min}$ was performed to yield single stranded DNA as a PCR template. Primers for each targeted loci containing adapters for Illumina sequencing were from IDT (Coralville, USA) (see Supplementary Table 1). PCR was done in a T100 Thermal Cycler (Bio-Rad) using the KAPA2G Robust PCR Kit (SIGMA, KK5024) with supplied buffer B and $3 \mu 1$ of cell extract in a total volume of $25 \mu 1$. The thermal cycling profile of the PCR was: $95^{\circ} \mathrm{C} 3 \mathrm{~min} ; 34 \mathrm{x}\left(95^{\circ} 15 \mathrm{sec}, 65^{\circ} \mathrm{C} 15 \mathrm{sec}, 72^{\circ} \mathrm{C} 15 \mathrm{sec}\right)$; $72^{\circ} \mathrm{C} 60$ sec. P5 and P7 Illumina adapters with sample specific indices were added in a second PCR reaction ${ }^{35}$ using Phusion HF MasterMix (Thermo Scientific, F-531L) and $0.3 \mu 1$ of the first PCR product. The thermal cycling profile of the PCR was: $98^{\circ} \mathrm{C} 30 \mathrm{sec} ; 25 \mathrm{x}\left(98^{\circ} 10 \mathrm{sec}, 58^{\circ} \mathrm{C} 10 \mathrm{sec}, 72^{\circ} \mathrm{C} 20 \mathrm{sec}\right)$; $72^{\circ} \mathrm{C} 5 \mathrm{~min}$. Amplifications were verified by size separating agarose gel electrophoresis using $2 \% \mathrm{EX}$ gels (Invitrogen, G4010-11). The indexed amplicons were purified using Solid Phase Reversible Immobilization (SPRI) beads in a 1:1 ratio of beads to PCR solution ${ }^{36}$. Double-indexed libraries were 
bioRxiv preprint doi: https://doi.org/10.1101/342311; this version posted June 8,2018 . The copyright holder for this preprint (which was not certified by peer review) is the author/funder, who has granted bioRxiv a license to display the preprint in perpetuity. It is made available under aCC-BY-NC-ND 4.0 International license.

sequenced on a MiSeq (Illumina) giving paired-end sequences of 2 x 150 bp ( $+7 \mathrm{bp}$ index). After base calling using Bustard (Illumina) adapters were trimmed using leeHom ${ }^{37}$.

\section{Sequencing data analysis}

Bam-files were demultiplexed and converted into .fastq files using SAMtools ${ }^{38}$. CRISPresso ${ }^{39}$ was used to analyse .fastq files for percentage of wildtype, HDR (any TNS), NHEJ (indels), and mix of HDR and NHEJ. Analysis was restricted to amplicons with a minimum of $70 \%$ similarity to the wildtype sequence and to a window of $20 \mathrm{bp}$ from each gRNA. Unexpected substitutions were ignored as sequencing putative errors. Sequencing data from colonies derived from single cell seeding (Fig. 3B and C) was analyzed using SAMtools and colonies were regarded as clones if the clear majority of reads consisted of a single sequence (homozygous) or of two sequences of similar read count (heterozygous) and blocking mutations, ancient mutations, as well as indels for each chromosome of the cells of a clone were noted (Supplementary Table 2).

\section{Karyotyping}

Microscopic analysis of the karyotype of 25 metaphases was done after trypsin induced Giemsa staining. The analysis was carried out in line with international quality guidelines (ISCN 2016: An International System for Human Cytogenetic Nomenclature ${ }^{40}$ ) by the 'Sächsischer Inkubator für klinische Translation' (Leipzig, Germany).

\section{Supplementary Data}

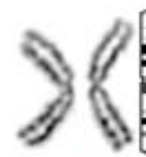

1

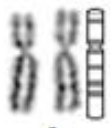

6

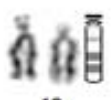

13

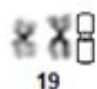

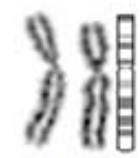

2

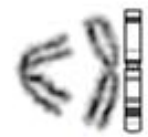

3

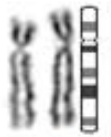

4
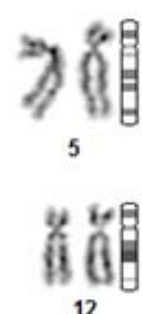

10

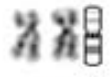

16

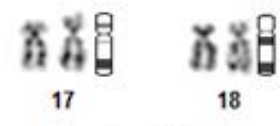

15

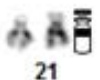

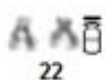

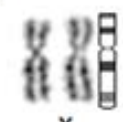

$\mathrm{X}$

Supplementary Fig. 1: Representative karyogram of 409-B2 iCRISPR hiPSCs with the DNA-PKcs KR mutation after 3 months in culture. Of 25 metaphases, analyzed by trypsin induced Giemsa staining, all show a healthy karyotype $(46, X X)$. No numerical or large scale chromosomal aberrations were identified (band number 350 , gray shades 3 ). 


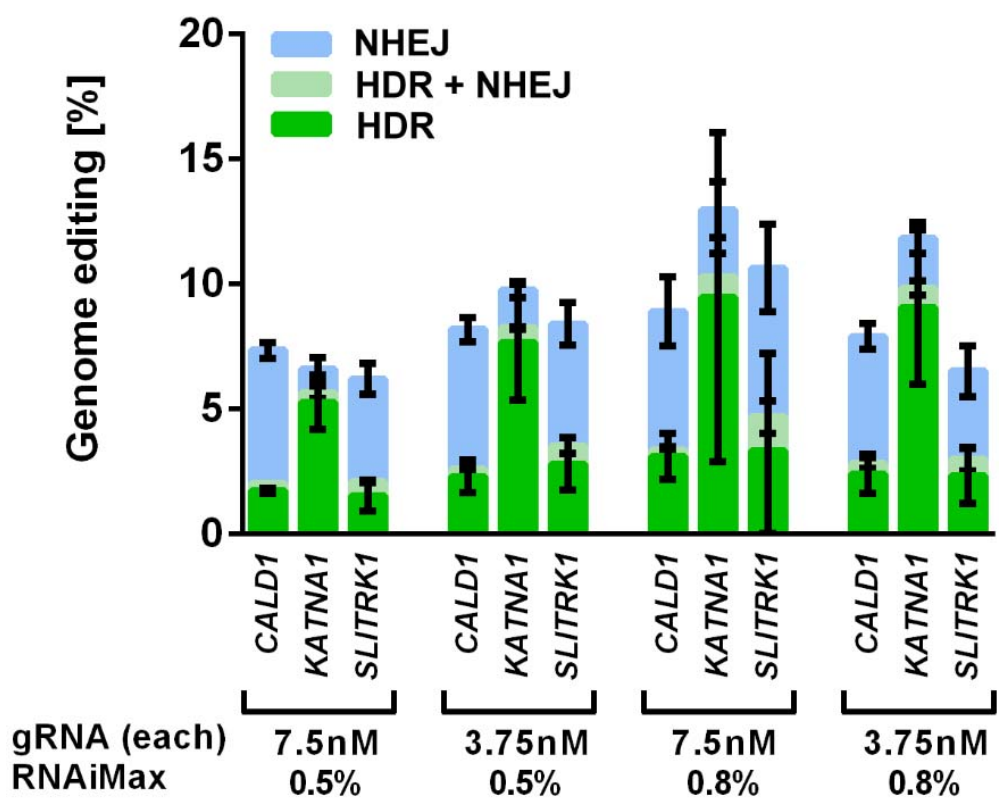

Supplementary Fig. 2: Genome editing efficiencies after multiplexed delivery of gRNAs and single stranded DNA donors of CALD1, KATNA1, and SLITRK1 by lipofection. Lipofection of gRNAs and single stranded DNA donors allows only low bulk HDR efficiencies for all three genes in 409-B2 hiPSCs with the DNA-PKcs KR mutation. Combinations of two gRNA and two lipofection reagent (RNAiMax) concentrations were tested. HDR, HDR + NHEJ, and NHEJ are shown in green, light green or blue, respectively. Error bars show the standard deviation of three technical replicates and cells were incubated for four days with doxycycline to express Cas9n for double nicking.

Supplementary Table 1: Oligonucleotides used. gRNA (crRNA target) and single stranded DNA donors (sSODNs) for editing of CALD1, KATNA1, SLITRK1, PRKDC, and HPRT, as well as primers for analysis and Q5 site-directed-mutagenesis of the Cas9 iCRISPR donor plasmid are shown. Mutations are in bold letters and ancestral mutations are underlined as well. The gRNA 'CALD1 t1' was used for Cas9n and Cas9 cleavage.

\begin{tabular}{|c|c|c|}
\hline \multirow{10}{*}{ gRNAs } & CALD1 t1 & TGGAGACTATTGCTGCTTGA \\
\hline & CALD1 t2 & GCAGTATACCAGTGCAATTG \\
\hline & KATNA1 $\mathrm{t} 1$ & AAATGATGACCCTTCCAAAA \\
\hline & KATNA1 t2 & CAACACCTAAAATAAGGGTA \\
\hline & SLITRK1 $\mathrm{t} 1$ & GCTAACAGTTTACCCTGCCC \\
\hline & SLITRK1 $\mathrm{t} 1$ & ACCCGTCGCTATCGCTGCTG \\
\hline & $P R K D C \mathrm{t} 1$ & GGTCCTCGCCACCCTTCACC \\
\hline & $P R K D C \mathrm{t} 2$ & GCGCGTGGAGCAGCTCTTCC \\
\hline & $P R K D C$ t1-back & GGTCCTCGCCACCTCTCACC \\
\hline & HPRT $\mathrm{t} 1$ & GGTTAAAGATGGTTAAATGAT \\
\hline \multirow{11}{*}{ ssODNs } & \multirow{2}{*}{ CALD1 Cas9 } & GTATACTGCTCCAGTCTGCTGTCAATCTTGGAGACTACTGCTGCTTGATGGGTCGATTTGACACCA \\
\hline & & CTGCTAAAAAAGTAAACACATACA \\
\hline & \multirow{3}{*}{ CALD1 Cas9n } & TTATATGTATGTGTTTACTTTTTTAGCAGTGGTGTCAAATCGACCCATCAAGCTGCAGTAGTCTCC \\
\hline & & AAGATTGACAGCAGACTGGAGCAGTATACCAGTGCTATTGAGGTGAGAATTGTCCTCAGCGTTATG \\
\hline & & GTCCTGCTGAACAGAAATAGA \\
\hline & \multirow{3}{*}{ KATNA1 Cas9n } & CTCATCTATATCCCAGGGAAAATTAGTAGCTGCCAGAACCATAACCATTTTAGAAGGGTCATCATT \\
\hline & & TTCAGAAGㅡACCTCCAACACCTAAAATAACGGTAAGGGGAGAGTGAAAAAGATATTAAGTTGGATT \\
\hline & & ATACCAAATGAAGCT \\
\hline & \multirow{3}{*}{ SLITRK1 Cas9n } & TCATCTTTAAACCCGACCCTGGGATGTGGTCGCAGCTGCAGCCCCCAGGGCATGGTAAACTGTTAG \\
\hline & & CTAAGGGTTTGTTCCTGGEGGTACCCGTCGCTATCGCAGCTGTGGGTCTGATTTTGATCTGCCAGT \\
\hline & & TGCCTGGGATCTTTGTACCTCCG \\
\hline
\end{tabular}


bioRxiv preprint doi: https://doi.org/10.1101/342311; this version posted June 8,2018 . The copyright holder for this preprint (which was not certified by peer review) is the author/funder, who has granted bioRxiv a license to display the preprint in perpetuity. It is made available under aCC-BY-NC-ND 4.0 International license.

\begin{tabular}{|c|c|c|}
\hline & \multirow{4}{*}{ PRKDC Cas9n } & \multirow[b]{2}{*}{ GCGAAGGCCCAAGCGCATCATCATCCGTGGCCATGACGAGAGGGAACACCCTTTCCTGGTGAGAGG } \\
\hline & & \\
\hline & & TGGCGAGGACCTGCGGCAGGACCAGCGCGTGGAGCAGCTCTTCCAGGTCATGAATGGGATCCTGGC \\
\hline & & CCAAG \\
\hline & & GCGAAGGCCCAAGCGCATCATCATCCGTGGCCATGACGAGAGGGAACACCCTTTCCTGGTGAAGGG \\
\hline & $P R K D C$-back Cas9n & TGGCGAGGACCTGCGGCAGGACCAGCGCGTGGAGCAGCTCTTCCAGGTCATGAATGGGATCCTGGC \\
\hline & & CCAAG \\
\hline & (1) Cnf1 & GCCATTTCACATAAAACTCTTTTAGGTTATAGATGGTTAAATGAATGACAAAAAAAGTAATTCACT \\
\hline & HPRI CPTI & TACAGTCTGGCTTATATCCAACAC \\
\hline \multirow{12}{*}{ Primers } & CALD1 forward & ACACTCTTTCCCTACACGACGCTCTTCCGATCTGCTAATCAGCTAGCATATGTATGAGAA \\
\hline & CALD1 reverse & GTGACTGGAGTTCAGACGTGTGCTCTTCCGATCTTTGGACTTGATTATTGTCCTAAGTG \\
\hline & KATNA1 forward & ACACTCTTTCCCTACACGACGCTCTTCCGATCTCCTGACGGCAAAGGAATATAG \\
\hline & KATNA1 reverse & GTGACTGGAGTTCAGACGTGTGCTCTTCCGATCTACTGTGCTTCCTTGTATTGTTGT \\
\hline & SLITRK1 forward & ACACTCTTTCCCTACACGACGCTCTTCCGATCTGGGCTTCAAATCAGCCAAG \\
\hline & SLITRK1 reverse & GTGACTGGAGTTCAGACGTGTGCTCTTCCGATCTTTTCAAGACAAATGGGCAAG \\
\hline & $P R K D C$ forward & ACACTCTTTCCCTACACGACGCTCTTCCGATCTCTAGCCTGTGCCCTGAGATG \\
\hline & $P R K D C$ reverse & GTGACTGGAGTTCAGACGTGTGCTCTTCCGATCTGCACAACGCTATAGGTCCTCA \\
\hline & HPRT forward & ACACTCTTTCCCTACACGACGCTCTTCCGATCTGGTGAAAAGGACCCCACGAA \\
\hline & HPRT reverse & GTGACTGGAGTTCAGACGTGTGCTCTTCCGATCTTGGCAAATGTGCCTCTCTACAAAT \\
\hline & Q5 D10A forward & TGGTGCCGATAGCCAGGCCGATG \\
\hline & Q5 D10A reverse & ACTCTGTGGGCTGGGCCG \\
\hline
\end{tabular}


bioRxiv preprint doi: https://doi.org/10.1101/342311; this version posted June 8,2018 . The copyright holder for this preprint (which was not certified by peer review) is the author/funder, who has granted bioRxiv a license to display the preprint in perpetuity. It is made available under aCC-BY-NC-ND 4.0 International license.

Supplementary Table 2: Overview of mutations introduced in CALD1, KATNA1, and SLITRK1 in 33 cellular clones. Integration of targeted nucleotide substitutions (left blocking mutation, 'ancient' missense mutation, and right blocking mutation) and insertion/deletions (indels) is labelled with ' $y$ ', while absence of these mutations is labelled with ' $n$ '. Homozygous (homo) or heterozygous (het) integration of the mutations is stated. ' $y^{*}$ ' indicates two cases where non-targeted missense nucleotide substitutions occur (clone 2 in CALD1 and clone 29 in KATNA1).

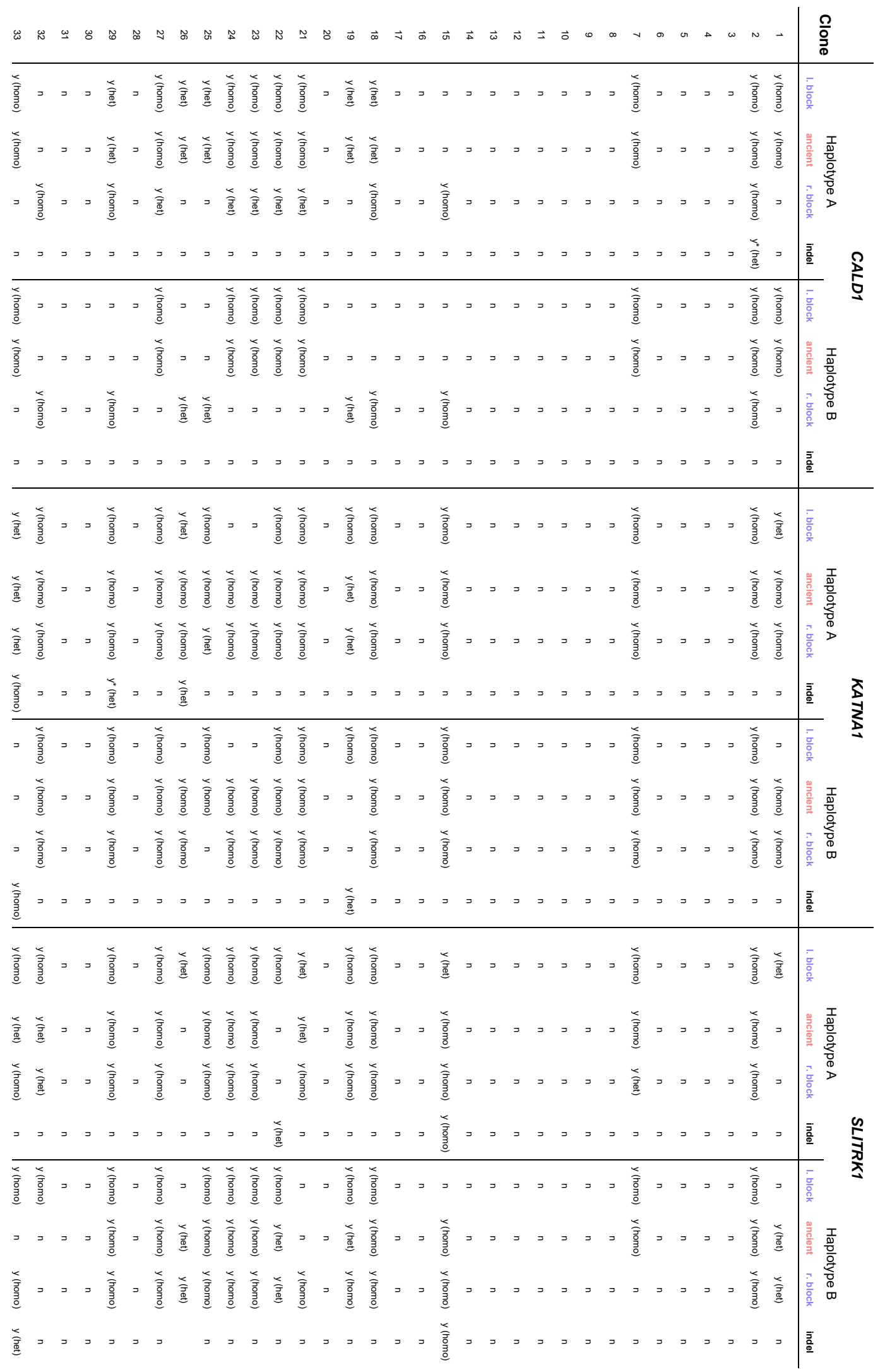




\section{Supplementary Material References}

34 Chari, R., Mali, P., Moosburner, M. \& Church, G. M. Unraveling CRISPR-Cas9 genome engineering parameters via a library-on-library approach. Nat Methods 12, 823-826, doi:10.1038/nmeth.3473 (2015).

35 Kircher, M., Sawyer, S. \& Meyer, M. Double indexing overcomes inaccuracies in multiplex sequencing on the Illumina platform. Nucleic Acids Res 40, e3, doi:10.1093/nar/gkr771 (2012).

36 Meyer, M. \& Kircher, M. Illumina sequencing library preparation for highly multiplexed target capture and sequencing. Cold Spring Harb Protoc 2010, pdb prot5448, doi:2010/6/pdb.prot5448 [pii]10.1101/pdb.prot5448 (2010).

37 Renaud, G., Stenzel, U. \& Kelso, J. leeHom: adaptor trimming and merging for Illumina sequencing reads. Nucleic Acids Res 42, e141, doi:10.1093/nar/gku699 (2014).

$38 \quad \mathrm{Li}, \mathrm{H}$. et al. The Sequence Alignment/Map format and SAMtools. Bioinformatics 25, 20782079, doi:10.1093/bioinformatics/btp352 (2009).

39 Pinello, L. et al. Analyzing CRISPR genome-editing experiments with CRISPResso. Nat Biotechnol 34, 695-697, doi:10.1038/nbt.3583 (2016).

40 International Standing Committee on Human Cytogenomic Nomenclature, McGowan-Jordan, J., Simons, A. \& Schmid, M. ISCN : an international system for human cytogenomic nomenclature (2016). (Karger, 2016). 